\title{
Análisis del conductismo, cognitivismo, constructivismo y su interrelación con el conectivismo en la educación postpandemia
}

\section{Analysis of behaviorism, cognitivism, constructivism and their interrelation with connectivism in post-pandemic education}

DOI: $10.46932 / \mathrm{sfjdv2n5-038}$

Received in: Oct 1st, 2021

Accepted in: Dec 30th, 2021

\author{
Marcel Oswaldo Méndez-Mantuano \\ Magíster en Gestión Ambiental \\ Instituto Superior Tecnológico Juan Bautista Aguirre \\ E-mail: marcelxc6768@hotmail.com \\ Evelyn Carolina Egüez Caviedes \\ Magíster en Dirección del Talento Humano \\ Instituto Superior Tecnológico Juan Bautista Aguirre \\ E-mail: evelyn.eguez@hotmail.com \\ Karla Vanessa Ochoa Ladines \\ Economista \\ Unidad Educativa Galo Plaza Lasso \\ Daule - Ecuador \\ E-mail: kava2020@hotmail.com \\ Danny Rafael Plúas Rogel \\ Maestro en Administración de la Educación \\ Unidad Educativa Galo Plaza Lasso \\ E-mail: danny_pluas00@hotmail.com \\ Carlos Enrique Paredes Yuqui \\ Economista \\ Unidad Educativa Galo Plaza Lasso \\ Daule - Ecuador \\ E-mail: ecoparedes@hotmail.com
}

\section{RESUMEN}

No son pocos quienes consideran que después de la pandemia por covid-19 el mundo debe enfrentarse a una nueva forma de cotidianidad, donde se evidenció que existen áreas que son indispensables para la subsistencia de la especie, mientras que otras pueden permitirse la transformación de sus espacios productivos para adaptarse a los requerimientos, quedando manifestó que el engranaje productivo-social puede regularse sin la excesiva burocratización de los procesos. Por otro lado, la educación también pudo desarrollarse dentro de las fronteras del hogar, sin la necesidad de acudir a los espacios educativos y esto solo fue posible por el desarrollo de las tecnologías de comunicación apropiadas. La adaptación rápida de las medidas educativas en ningún caso supone "innovación", simplemente fueron la respuesta resiliente propia de las personas, por lo tanto, debemos interiorizar si los resultados que se lograron durante el desarrollo de la virtualidad permitieron el correcto aprendizaje de los estudiantes dentro de sus hogares. 
Dentro de este último aspecto, se asume que existirán muchas investigaciones que abordarán dicho análisis, por lo cual, la presente investigación tiene como propósito establecer las diferencias y similitudes entre varias teorías de enseñanza-aprendizaje, tales como el conductismo, el cognitivismo y el constructivismo, para posteriormente determinar una simbiosis teórica que podrían desarrollar estas teorías de enseñanza-aprendizaje dentro de la estructura de la educativa postpandemia. A criterio de los autores se seleccionaron estas tres teorías, ya que se consideran que son las que actualmente se desarrollan dentro de los diferentes escenarios pedagógicos. Para esto, se realizó una revisión bibliográfica de diferentes fuentes especializadas, para conocer los fundamentos conceptuales sobre los cuales se desarrollan las teorías antes mencionadas. Posteriormente, se realizaron esquemas que permitieron la priorización de los ejes. Entre las principales características se encuentra que el conductismo estudia de forma objetiva la conducta que desarrollan las personas, por su parte, el cognitivismo concibe que los aprendizajes y conocimientos se originan desde la experiencia que cada uno desarrolla con el entorno, mientras que el constructivismo intenta explicar la naturaleza de los conocimientos humanos. Finalmente se trata de examinar al conectivismo como una teoría emergente, sin embargo, se denota que la misma carece de los fundamentos apropiados para ser considerada como un nuevo paradigma educativo. Se concluye que la neoeducación debe poseer componentes moldeables, que permitan cambios en el proceso de enseñanza-aprendizaje a la misma velocidad que lo realiza la tecnología o la sociedad.

Palabras claves: cognitivismo, conductismo, conectivismo, constructivismo, nuevo paradigma, postpandemia, teorías educativas

\begin{abstract}
There are few who consider that after the COVID-19 pandemic the world must face a new form of daily life, where it was evidenced that there are areas that are essential for the subsistence of the species, while others can afford the transformation of their productive spaces to adapt to the requirements, being stated that the productive-social gear can be regulated without excessive bureaucratization of the processes. On the other hand, education could also be developed within the borders of the home, without the need to go to educational spaces and this was only possible due to the development of appropriate communication technologies. The rapid adaptation of educational measures in no case implies "innovation", they were simply the resilient response of the people, therefore, we must internalize if the results that were achieved during the development of virtuality allowed the correct learning of the students within their homes. Within this last aspect, it is assumed that there will be many investigations that will address this analysis, therefore, the present investigation aims to establish the differences and similarities between various teaching-learning theories, such as behaviorism, cognitivism, and constructivism, to later determine a theoretical symbiosis that these teaching-learning theories could develop within the post-pandemic educational structure. At the discretion of the authors, these three theories were selected, since they are the ones currently being developed within the different pedagogical settings. For this, a bibliographic review of different specialized sources was carried out, to know the conceptual foundations on which the theories are developed. Subsequently, schemes were made that allowed the prioritization of the axes. Among the main characteristics is that behaviorism objectively studies the behavior that people develop, for its part, cognitivism conceives that learning and knowledge originate from the experience that each one develops with the environment, while constructivism tries to explain the nature of human knowledge. Finally, it is about examining connectivism as an emerging theory, however, it is noted that it lacks the appropriate foundations to be considered as a new educational paradigm. It is concluded that neoeducation must have moldable components that allow changes in the teaching-learning process at the same speed as technology or society does.
\end{abstract}

Keywords: cognitivism, behaviorism, connectivism, constructivism, new paradigm, post-pandemic, educational theories 


\section{INTRODUCCIÓN}

La importancia de analizar las teorías educativas radica en que estas permiten conocer los postulados básicos sobre el proceso de enseñanza-aprendizaje entre los alumnos y sus docentes. La anterior interacción permite que los conocimientos se sigan transfiriendo de una generación a otra, donde la acumulación entre los conocimientos pasados y presentes permite el desarrollo de todos los ámbitos sociales y productivos.

Los agentes educativos consideran que el rol de la enseñanza se encuentra en la humanización de las personas y en la transformación social del planeta. Por ende, las teorías educativas tratan de determinar los protocolos en la formación integral de los sujetos, por medio del impulso de sus habilidades, para convertirlo en actor principal de su propia historia y no en un objeto de ella (Gadotti, 2003).

Las teorías en la educación son creadas desde una visión particular, con el objetivo de intentar dar respuesta a una determinada problemática. Nacen desde la construcción dialéctica de una hipótesis, la misma que se desarrolla dentro de una base empírica que muchas veces no representa de manera congruente a la realidad escolar, por lo tanto, estas teorías se convierten en reflexiones apócrifas que luego se modulan como pedagogías normativas. Esto denota que no toda teoría educativa es pertinente dentro de una determinada temporalidad o espacialidad (Narvaja \& Andrea, 2018).

Desde el siglo XX las ciencias pedagógicas vienen debilitándose en el mundo entero, las cuales, no son vistas como ciencias educativas por excelencia y se cuestiona de manera repetida su rol dentro de la sociedad. Estos debates se centran en las siguientes premisas: la pedagogía como ciencia autónoma, problemas derivados de la epistemología pedagógica, la pedagogía como dependiente de las ciencias de la educación (Cánovas \& Chávez, 2020). Las teorías educativas son modelos que se llevan a cabo dentro del proceso de aprendizaje, donde cada una tiene sus propios espacios conceptuales para su aplicación, por lo tanto, existen diferentes niveles de validez y su efectividad específica no puede ser generalizada, sino que varía por diferentes factores presentes (Figueroa, Muñoz, Lozano, \& Zavala, 2017).

No son pocos quienes consideran que después de la pandemia por covid-19 el mundo debe enfrentarse a una nueva forma de cotidianidad, donde se evidenció que existen áreas que son indispensables para la subsistencia de la especie, mientras que otras pueden permitirse la transformación de sus espacios productivos para adaptarse a los requerimientos, quedando manifestó que el engranaje productivo-social puede regularse sin la excesiva burocratización de los procesos. Por otro lado, la educación también pudo desarrollarse dentro de las fronteras del hogar, sin la necesidad de acudir a los espacios educativos y esto solo fue posible por el desarrollo de las tecnologías de comunicación apropiadas. 
La adaptación rápida de las medidas educativas en ningún caso supone “innovación”, simplemente fueron la respuesta resiliente propia de las personas, por lo tanto, debemos interiorizar si los resultados que se lograron durante el desarrollo de la virtualidad permitieron el correcto aprendizaje de los estudiantes dentro de sus hogares. Dentro de este último aspecto, se asume que existirán muchas investigaciones que abordarán dicho análisis, por lo cual, la presente investigación tiene como propósito establecer las diferencias y similitudes entre varias teorías de enseñanza-aprendizaje, tales como el conductismo, el cognitivismo y el constructivismo, para posteriormente determinar una simbiosis teórica que podrían desarrollar estas teorías de enseñanza-aprendizaje dentro de la estructura de la educativa postpandemia. A criterio de los autores se seleccionaron estas tres teorías, ya que se consideran que son las que actualmente se desarrollan dentro de los diferentes escenarios pedagógicos.

\section{METODOLOGÍA}

La investigación tiene un enfoque cualitativo, debido a que se establecieron las diferencias y similitudes conceptuales que existen entre el conductivismo, cognitivismo y constructivismo. Luego se establecieron los puntos convergentes en los cuales las teorías educativas antes descritas pueden colaborar en la educación postpandemia. Así mismo, se analizaron las posturas conceptuales que aborda el actual conectivismo, lo que permitió analizar si este puede considerarse como una nueva tendencia educativa o solo una reformulación de los postulados clásicos.

Posee un diseño de tipo documental, ya que las premisas abordadas de las diferentes teorías de la educación fueron recopiladas de documentos que principalmente se encuentran disponibles en la web. Estos documentos fueron seleccionados por su rigurosidad en el análisis y por su soporte en las teorías clásicas.

Finalmente, la investigación posee un alcance descriptivo, donde únicamente se analizan, caracterizan y clasifican las teorías, con el objetivo de esclarecer los objetivos teóricos que persiguen cada una de ellas, para así, contrastarlos con los objetivos del conectivismo y con ello poder examinar si este es el nuevo orbe sobre el cual se fundamenta la educación que emerge después de la pandemia.

\section{DESARROLLO}

3.1 NOCIONES, SIMILITUDES Y DIFERENCIAS ENTRE CONDUCTISMO, COGNITIVISMO Y CONSTRUCTIVISMO

A diferencia de los que muchos textos consideran, el conductismo no apareció en la palestra de la Psicología de manera abrupta ni inesperada, sino que tuvo un desarrollo progresivo, tal como sucede en la mayoría de las ciencias sociales. En inicios del siglo XX, el idealismo filosófico era reemplazado por 
las nuevas formas de pragmatismo, de realismo y del instrumentalismo científico; además el desarrollo de la Psicología, de la teoría evolutiva y la sustitución de la reproducción de los conocimientos por el desarrollo del pensamiento relacional, permitieron que surgiera el conductismo. El mismo que fue reinterpretado de manera asertiva por Watson en 1913, quien actuó como mánager artístico de este paradigma (Peña, 2011).

Dentro del conductismo la conducta es definida como las acciones que realizan los individuos de manera individual o colectiva. Cabe mencionar que existen varias nociones sobre lo que es la conducta, sin embargo, existen acuerdos para considerar que esta es un fenómeno dinámico que se desarrolla durante todo el tiempo de vida de un organismo. Así mismo, dentro del conductismo el aprendizaje es un resultado de la interacción con la realidad, son temporales y los conocimientos que son aprendidos pueden ser desaprendidos (Peña, 2011).

Según Hergenhahn (2011), los ejes principales sobre los cuales gira el conductismo son los siguientes:

- La conducta de las personas es aprendida e influida por el medio, por lo tanto, los rasgos genéticos tienen poca influencia en el desarrollo conductual.

- El lenguaje es una manifestación de la conducta determinada por los principios del aprendizaje.

- Los procesos de aprendizaje de los humanos y animales son parecidos, por lo tanto, estudiar a los últimos puede desvelar los procesos llevados a cabo en las personas.

- Los procesos que se desarrollan dentro de la mente pueden ser minimizados por las personas.

- Las respuestas a ciertos estímulos pueden ser modificadas con un cambio en la aplicación de los principios de los aprendizajes.

- Los principios de aprendizaje son los mismos para un individuo joven o adulto.

Entre las principales críticas que se realizan al conductismo se encuentran las siguientes (Soriano, 2011):

- Ignora la influencia que tiene el inconsciente.

- No desarrolla una explicación para los procesos cognoscitivos (intuición, creatividad, imaginación).

- Es mecanicista (todo lo resumen en estímulo-respuesta).

- Está desarticulada con las ciencias modernas (estudia animales para intentar predecir el comportamiento humano).

- Es operacionalista, ya que se encuentra al servicio del sistema y concibe a la Psicología como ciencia naturalista y no social.

Los fundamentos del cognitivismo fueron establecidos por Piaget y Vygotsky, quienes interpretaron que el aprendizaje en los niños se realizaba a diferentes edades y etapas, además mencionaron que los procesos sociales tienen influencia en el desarrollo de las habilidades intelectuales de las personas (Linares, 2007). Pensadores de la década del 50 como Herbert Simon, Noam Chomsky y Marvin Minsky se transforman en cofundadores de las bases cognitivistas, los mismos que indican que la 
inteligencia y la intencionalidad se forman en las representaciones que adquieren la realidad a través de los códigos simbólicos que interpreta el cerebro (Bächler, 2014).

El cognitivismo considera que las funciones invariables del aprendizaje son la organización y la adaptación, el primero indica que los niños a medida que se desarrollan integran patrones físicos o mentales simples y los convierten en sistemas complejos, mientras que el segundo término hace referencia a la capacidad innata de moldear las estructuras de acuerdo con los estímulos recibidos por el mundo exterior. También la asimilación es el proceso mental en la que encaja la información recibida de acuerdo con patrones preestablecidos. Cuando la información es incompatible se realiza el proceso de acomodación, el cual ayuda a integrar los elementos nuevos con los preexistentes. Estos dos últimos procesos cognitivos se encuentran interrelacionados y explicarían los cambios en los conocimientos que se producen durante la vida de un individuo (Linares, 2007).

Para Piaget, existen cuatro factores que intervienen en los procesos cognitivos, los cuales son:

- Maduración o desarrollo de las estructuras físicas y mentales que fueron heredadas de los padres a los hijos.

- Interrelación física de los sujetos con el entorno que los rodea.

- Transmisión de información y de conocimientos que realiza la sociedad a los sujetos.

- Establecimiento del equilibrio a través de los procesos de asimilación y acomodación.

Piaget también indicó que existen al menos cuatro etapas en el desarrollo cognitivo de los niños: etapa sensoriomotora, etapa preoperacional, etapa de operaciones concretas y etapa de operaciones formales.

Según Pons (2013), entre los principales escollos que se mencionan en el cognitivismo se destacan los siguientes:

- Excesivo racionalismo en la noción de ser humano: la persona es concebida por el cognitivismo como una "máquina de pensar"

- Las perspectivas no son sociales, las teorías analizan el conocimiento individual de las personas y no la construcción de este como un factor social

- Desinterés por los aspectos emocionales y motivacionales, donde el computador se transforma en un buen modelo para explicar la actividad de la mente humana.

- Los procesos humanos son percibidos desde una visión mecanicista, donde se priorizan las operaciones mentales formales y se relegan las dinámicas sociales.

- Los postulados esgrimidos dentro del cognitivismo no son objetivos, donde se la eleva a la conclusión incuestionable de todos los paradigmas mentales de las personas.

El constructivismo tuvo su inspiración en las teorías genéticas de Jean Peaget, esta teoría también adopta sus fundamentos en el aprendizaje verbal, los organizadores previos y en la asimilación. Los primeros trabajos en esta área fueron realizados por Vygotski en los años 30 y se enriquecieron por los aportes de Ausubel, Novak y Gowin en los años 50 y 60. Para algunos autores, el constructivismo es el 
rediseño de la Psicología cognitiva, donde toma los enfoques del procesamiento de la información (Coll, 1996).

El constructivismo es la edificación de los aprendizajes de parte de sujeto activo, donde se involucran elementos personales materiales (físicos) e inmateriales (mentales). Por lo tanto, el conocimiento que se genera no es una fiel copia de la realidad, sino una construcción que se origina de la dialéctica con el entorno que lo rodea, ergo cada persona es capaz de formar su propia representación del mundo real de acuerdo con sus motivaciones y experiencias (Berrocal, 2013).

Para Ertmer \& Newby (1993), los supuestos en los cuales se fundamenta el constructivismo son los siguientes:

- Énfasis en la identificación del contexto en el cual las habilidades serán aprendidas y luego aplicadas, así los aprendizajes se darán en contextos significativos.

- Capacidad para que el estudiante manipule la información y realice su propio control, así el estudiante utilizará lo que él realmente aprende.

- La necesidad de que la información se presente en una amplia variedad de formas, en contenidos en distintos momentos, en contextos reestructurados, para propósitos diferentes y desde diferentes perspectivas conceptuales.

- Apoyar el uso de las habilidades de solución de problemas que permitan al estudiante ir más allá de la información presentada, a través de diferentes formas de presentar problemas.

- La evaluación debe estar dirigida hacia la transferencia de conocimiento y habilidades, a través de la presentación de problemas que difieran de las condiciones de la instrucción inicial

Para Barreto, Gutiérrez, Pinilla, \& Parra (2006), las críticas que principalmente se enuncian para el constructivismo son:

- Los supuestos son a veces considerados como verdades absolutas, esto genera que se omitan vacíos sobre los procesos constructivos del pensamiento.

- No abordan el cúmulo de dimensiones que encauzan al sujeto, por lo tanto, no se exploran todas las potencialidades que pueden desarrollar las personas.

- Los aspectos motivacionales y afectivos han sido relegados a segundo plano, ya que no se clarifica la función que estos desempeñan dentro de los procesos de enseñanza y aprendizaje.

- Su excesivo corte racional le dan características propias de una forma de instrucción mecanicista y no formativa, con énfasis en las particularidades receptivas y estáticas.

- Los planteamientos constructivistas se limitan a dar explicaciones de cómo los estudiantes se van asemejando a su profesor, donde se omiten las acciones individuales de los estudiantes.

A continuación, se bosqueja el siguiente cuadro comparativo de los paradigmas educativos analizados: 
Tabla 1. Comparación entre conductismo, cognitivismo y constructivismo. Fuente: Valdez (2012). Elaboración: propia

\begin{tabular}{|c|c|c|c|}
\hline Teoría & Conductismo & Cognitivismo & Constructivismo \\
\hline Características & $\begin{array}{l}\text { Estudia de forma } \\
\text { objetiva la conducta } \\
\text { que desarrollan las } \\
\text { personas }\end{array}$ & $\begin{array}{lr}\text { Concibe que } & \text { los } \\
\text { aprendizajes } & \text { y } \\
\text { conocimientos } & \text { se } \\
\text { originan desde } & \text { la } \\
\text { experiencia que cada } \\
\text { uno desarrolla con el } \\
\text { entorno }\end{array}$ & $\begin{array}{l}\text { Intenta explicar la } \\
\text { naturaleza de los } \\
\text { conocimientos } \\
\text { humanos }\end{array}$ \\
\hline Objetivos formativos & $\begin{array}{l}\text { Lograr respuestas } \\
\text { óptimas de los } \\
\text { estudiantes frente a los } \\
\text { estímulos apropiados }\end{array}$ & $\begin{array}{lr}\text { Estimulación } & \text { de } \\
\text { estrategia } & \text { de } \\
\text { aprendizaje por } & \text { parte } \\
\text { del alumno } & \end{array}$ & $\begin{array}{l}\text { Los aprendizajes } \\
\text { forman parte de un } \\
\text { proceso participativo } \\
\text { entre estudiantes y } \\
\text { profesores }\end{array}$ \\
\hline $\begin{array}{l}\text { Función de los } \\
\text { estudiantes }\end{array}$ & $\begin{array}{l}\text { Obediente a los } \\
\text { procesos educativos }\end{array}$ & $\begin{array}{l}\text { Participa en el proceso } \\
\text { de enseñanza- } \\
\text { aprendizaje }\end{array}$ & $\begin{array}{l}\text { Es responsable de la } \\
\text { generación } \\
\text { conocimientos en el } \\
\text { proceso de enseñanza- } \\
\text { aprendizaje }\end{array}$ \\
\hline $\begin{array}{c}\text { Función de los } \\
\text { docentes }\end{array}$ & $\begin{array}{l}\text { Diseña y controla los } \\
\text { estímulos }\end{array}$ & $\begin{array}{l}\text { Adapta las } \begin{array}{l}\text { estrategias } \\
\text { de } \\
\text { enseñanza- } \\
\text { aprendizaje }\end{array} \\
\end{array}$ & $\begin{array}{l}\text { Es un tutor en el } \\
\text { proceso de enseñanza- } \\
\text { aprendizaje }\end{array}$ \\
\hline Criterios de evaluaci & Cuantitativa & $\begin{array}{l}\text { Centralizada en los } \\
\text { procesos }\end{array}$ & Permanente \\
\hline
\end{tabular}

\subsection{CONDUCTISMO, COGNITIVISMO Y CONSTRUCTIVISMO EN LA EDUCACIÓN} POSTPANDEMIA

Es impreciso indicar que dentro de la educación actual no se aplica uno de los paradigmas (conductismo, cognitivismo y constructivismo), o que uno de ellos se encuentra en desuso o sea considerado obsoleto, tal como es el caso del conductismo. Tampoco se puede negar que existen predilecciones de parte del sistema educativo "moderno" por aplicar herramientas constructivistas dentro de las aulas. Estos fenómenos no son nuevos, sino que la historia demuestra que existen ciclos de preferencias dentro de una determinada época, lo que fue una tendencia hace décadas, hoy puede ser visto como algo rutinario, simplista o antipedagógico. No se niega la evolución que han desarrollado los diferentes paradigmas, lo que a continuación se intenta es demostrar que los tres paradigmas se encuentran tan vigentes como en antaño, pero que los mismo no son identificados de manera correcta ya que sus prácticas han evolucionado de forma constante, pero los principios en los cuales se basa su funcionamiento siguen indemnes.

La teoría conductista considera que la conducta de una persona es aprendida bajo un sistema de refuerzos (positivos o negativos) y castigos desde el nacimiento del individuo. Estas premisas psicológicas fueron llevadas al campo educativo, donde en el pasado se llegaba a infligir castigos físicos a los alumnos para intentar excluir los "errores" que estos realizaban dentro del proceso de enseñanza-aprendizaje. El mismo conductismo bosquejó lo denominado como "condicionamiento operante", el cual indica que el comportamiento de una persona se repetirá de manera secuencial en función de los premios o castigos que 
se le den a un determinado comportamiento, donde se pueden obtener cuatro tipos de respuestas: castigos positivos y negativos, y refuerzos positivos y negativos. Según estudios, los castigos muestran ser más efectivos que los refuerzos al momento de intentar modificar una determinada conducta. Por ello, los docentes en la actualidad "ignoran" los comportamientos negativos de sus estudiantes y "premian" las conductas consideradas como aceptables, otro ejemplo de conductismo en el aula es el sistema de calificaciones basado en notas, donde existen rangos para determinar la aprobación o reprobación de los estudiantes (Rodríguez, 2019).

El cognitivismo estima que los procesos mentales y la generación del conocimiento se encuentran por encima de la simplificación dada por los estímulos y respuestas. Dentro del cognitivismo se enfatiza en los procesos que generan los conocimientos por encima de las respuestas que se den, además los individuos son pensados como estructuras activas complejas capaces de generar sus propias respuestas de acuerdo con sus motivaciones individuales y no como escuetos depósitos pasivos que reaccionan a los diferentes estímulos del entorno. El cognitivismo nació de las pesquisas surgidas para entender los procesos mentales de los seres humanos, para intentar reproducirlos dentro de los ordenadores que surgieron a partir de la década de los 60. El cognitivismo dentro del aula se lo evidencia cuando el estudiante genera su aprendizaje de manera autónoma a partir de la codificación y almacenamiento de información nueva (proveniente de varios medios), donde todo el proceso está enfocado al razonamiento, la resolución de los problemas presentados y a la adaptación posible de los resultados, por ejemplo, cuando los docentes exponen los aspectos generales de la clase y posteriormente se motiva a los estudiantes a realizar determinados trabajos (Chávez, Chávez, Padrón, \& Martínez, 2009).

Por su parte, el constructivismo considera que los aspectos sociales y cognitivos del comportamiento en las personas es una construcción permanente y no un resultado mecánico de la simple interacción con el ambiente o de sus motivaciones internas, ergo el conocimiento no es una copia de la realidad, sino una construcción mental y particular de los seres humanos. Esta representación de la realidad depende de factores internos como externos, además de los elementos que refuerzan el aprendizaje. Es posible comparar la construcción de los conocimientos con un trabajo mecánico, ya que los esquemas que se generan en el interior del cerebro son como herramientas específicas que permiten la realización de una determinada actividad, pero que cumplida dicha función no es útil para las consiguientes. Un esquema es una representación situacional específica que son manejados internamente y confrontados con el entorno, esto implica que las personas no actúan directamente sobre la realidad, sino que lo realizan a través de esquema preexistentes derivados de procesos anteriores (sociales, cognitivos, internos, externos, etc.). Esta interacción constante también moldea los esquemas, es decir, entre más experiencia se posea en una determinada tarea, las personas usarán esquemas más complejos 
que les permitan interactuar de manera diferente cada vez que realicen la misma tarea (Carretero, 2005). Dentro del aula existen varias escenas donde se puede identificar el constructivismo, por ejemplo, explicar ciertas nociones del funcionamiento particular de una cosa material y solicitarles a los estudiantes construir algo análogo, esto genera que se aprenda a través de las experiencias previas y permite erigir nuevos contextos a partir de escenarios holísticos dentro del aula.

Todo lo anterior respalda que las tres teorías de enseñanza-aprendizaje siguen presentes dentro de las aulas latinoamericanas en menor o mayor medida (inferencia derivada de los paralelismos socioculturales presentes en los países latinoamericanos). Este análisis no tiene como finalidad realizar un juicio de valor sobre que es mejor o peor, sino mostrar a los docentes que herramientas seguimos utilizando, y cómo a través de una interiorización podemos mejorar los resultados académicos obtenidos hasta el día de hoy en cada una de nuestras aulas.

En otro aspecto, pocos se arriesgan a predecir que los elementos trastocados por la pandemia del 2020 serán similares a estas fechas, incluso se habla de la adaptación de la sociedad a una "nueva realidad”. A partir de ello, surge la pregunta ¿Cómo se debe desarrollar la educación postpandemia?, no existe una respuesta clara para abordar esta disyuntiva, pero se conocen los siguientes aspectos:

- La educación no será igual a los años previos del 2020,

- La educación debe migrar hacia nuevos paradigmas que integren la tecnología,

- El sistema productivo exige resultados al sistema educativo a corto plazo,

- El desarrollo de la globoeducación se potenciará en varios países,

- La humanidad debe considerar que no será la última pandemia que deberá enfrentar,

- Entre otros aspectos.

Quizás nos encontremos en un momento álgido de la historia educativa, donde se surca por un puente sinuoso que nos direcciona hacia el desarrollo de una nueva teoría educativa, la cual tendrá que responder a los nuevos retos contemporáneos. O quizás nos encontramos frente a una nueva evolución de los tradicionales paradigmas. No existe una forma de establecer si nos encontramos frente a un nuevo hito histórico mientras que se desarrolle el mismo, sin embargo, a continuación, se abordarán los nuevos postulados que están en desarrollo, para determinar si los mismos son paralelismos conceptuales a los paradigmas tradicionales o realmente nos encontramos frente a una nueva área del aprendizaje educativo. Solo la prueba del tiempo lo determinará.

Las tres grandes teorías de aprendizajes o paradigmas ante mencionados son las más usadas en la creación de los ambientes educativos, sin embargo, estas fueron planteadas en unas épocas muy diferentes en donde no existía una alta conectividad, la globalización era vista como una utopía, y los retos sociales, ambientales y económicos eran abordados desde una dimensionalidad local y no a nivel planetaria. Con estas nuevas premisas nace el concepto de conectivismo, el cual integra de manera activa a las Tecnologías 
de la Información y Comunicación (TIC) dentro todo el proceso de enseñanza y aprendizaje (Tumino \& Bournissen, 2016).

El conectivismo fue desarrollado principalmente por George Siemens, quien expresó que esta teoría del aprendizaje es un producto casi natural de la era digital, la misma que trata de analizar el aprendizaje complejo dentro de un orden social moderno que cambia de manera rápida y constante. La propuesta define que el aprendizaje se lo realice a través de redes las cuales poseen nodos y conexiones internas. Según el autor el conectivismo es un proceso que ocurre exclusivamente dentro de la infraestructura de los entornos virtuales de enseñanza-aprendizaje, no es una actividad individual, sino una interacción entre los aprendices y el ecosistema digital. La teoría indica que las decisiones de los individuos se fundamentan en la transformación permanente que sufren las bases, ya que de manera continua la nueva información adquirida deja obsoleta la información anterior. Los principios que fundamental la teoría conectivista son los siguientes (Siemens, 2005):

- El aprendizaje y el conocimiento se fundamentan en la pluralidad de opiniones de los individuos.

- El aprendizaje es un proceso conectivo entre nodos especializados, también denominados fuentes de información.

- El aprendizaje también se aloja en los dispositivos tecnológicos.

- La capacidad para obtener más conocimiento es más importante de lo que se considera.

- Promover y conservar las conexiones es un mecanismo vital para proporcionar un aprendizaje continuo.

- La capacidad para establecer conexiones entre los campos, las ideas y los conceptos es fundamental en el proceso de enseñanza-aprendizaje.

- Dentro del aprendizaje conectivista se prepondera que los conocimientos se encuentren de manera exacta y actualizados.

- Tomar decisiones forma parte del proceso de aprendizaje, ya que la realidad es cambiante y esto modifica el clima de la información que se posee, lo cual afecta a la toma de las decisiones.

También se menciona que los actuales sistemas educativos deben transformarse en sociedades del aprendizaje, ya que el mundo contemporáneo se encuentra altamente conectado y en constantes cambios. En el pasado, dentro de las formas de aprendizaje primaba la competencia y represión, mientras que los nuevos valores educativos deben fomentar la colaboración integral entre personas sin ningún tipo de sesgos (CISCO, 2010).

También existen varios detractores para esta teoría, entre ellos Zapata-Ros (2014), quien menciona que el conectivismo no posee elementos fundamentales para ser considerada como una teoría educativa, por ejemplo, no posee objetivos claramente definidos, no posee valores estructurados, no se establecen las condiciones de aplicación, no existe una metodología, no posee todos los elementos de que constan las teorías, no existe una forma de validación, además los problemas son abiertos y las líneas de desarrollo futuras no se encuentran plenamente establecidas. Ya que carece de los elementos antes indicados se la ha catalogado como una perspectiva pedagógica y no como una teoría debidamente fundamentada (Pløn 
Verhagen, 2006). También se indica que los principios del conectivismo no se encuentran suficientemente vinculados a los argumentos ni a los ejemplos presentados por Siemens, por lo tanto, es difícil estructurar un sistema de ideas sobre el funcionamiento práctico de la teoría (Zapata-Ros, 2014).

El conectivismo no logra superar los problemas de las otras teorías educativas existentes, incluso carece de los sustentos necesarios para denominarse teoría educativa. Por lo tanto, regresamos a la premisa original, ¿cuál debe ser el rol de los tres grandes paradigmas en la educación postpandemia?

Al interiorizar todos los preceptos mencionados, se puede indicar que la neoeducación postpandemia debe tener las siguientes características:

- Que permita la flexibilidad a las particularidades de los diferentes estudiantes.

- Que sea inclusiva con las TIC.

- Que permita la posibilidad de realizarse de manera remota.

- Que rediseñe el sistema de evaluación, el cual se encuentre orientado en el proceso y no en los resultados.

- Que fomente la participación de todo el ecosistema escolar (interno y externo).

- Que permita la realización de cambios en la metodología de manera rápida.

- Que prepondere la práctica sobre la teoría.

- Que propicie la reflexión y la autocrítica.

- Que estimule los sentidos desde las edades tempranas.

\section{CONCLUSIONES}

El conductismo analiza de forma objetiva el comportamiento conductual que desarrollan las personas, el mismo que se basa en estímulos y respuestas. Por su parte, el cognitivismo considera que los aprendizajes y conocimientos son desarrollados por la experiencia individual de las personas con su entorno. Mientras que el constructivismo integra elementos materiales o físicos e inmateriales o mentales dentro del proceso de enseñanza y aprendizaje. Estas tres teorías siguen vigentes dentro de las funciones educativas, donde se usan recursos cognitivos y constructivos en el desarrollo de las actividades áulicas y el sistema de evaluación mantiene el esquema conductista del estímulo-respuesta.

El conectivismo carece de los fundamentos elementales para ser considerado como otro gran paradigma dentro del orbe educativo, sin embargo, sus principios pueden ser integrados en el desarrollo de una neoeducación que acompañe de manera distinta a los estudiantes en la nueva realidad postpandemia. Esta evolución educativa debe integrar elementos líquidos (moldeables) que permitan la flexibilidad en aspectos como la forma de enseñar, el lugar que se enseña, el lugar desde que se aprende, los recursos necesarios para todo el proceso, entre otros. No existe un modelo ideal, ya que no existen personas ideales, nuestros productos son el fiel reflejo de una sociedad que cambia de acuerdo con los retos presentes, por lo tanto, la educación seguirá la constante transformación de sus principios hasta que el mundo deje de existir o los humanos dejemos de aprender, seguro pasará lo primero, ya que lo segundo es parte de nuestra propia naturaleza. 


\section{BIBLIOGRAFÍA}

Bächler, R. (2014). El cognitivismo y la negación de la mente: influencia del dualismo cartesiano. Panorama, 8(14), 47-58. Obtenido de https://dialnet.unirioja.es/servlet/articulo?codigo=4799370

Barreto, C., Gutiérrez, L., Pinilla, B., \& Parra, C. (2006). Límites del constructivismo pedagógico. Educación y $\quad$ Educadores, 9(1), Obtenido 11-31. de http://www.scielo.org.co/scielo.php?script=sci_arttext\&pid=S0123-12942006000100002

Berrocal, D. (2013). Análisis crítico de la pedagogía constructivista. Investigación Educativa, 17(2), 97104.

Cánovas, L., \& Chávez, J. (2020). Compendio de pedagogía. La Habana: Pueblo y Educación.

Carretero, M. (2005). ¿Qué es el constructivismo? México D.F.: Progreso.

Chávez, I., Chávez, M., Padrón, M., \& Martínez, H. (2009). Conductismo, Cognitivismo y Diseño Instruccional. Obtenido de https://issuu.com/jheiseo/docs/conductismo_cognitivismo_y_dise_o

CISCO. (2010). The Learning Society. Obtenido - de https://www.cisco.com/c/dam/en_us/about/citizenship/socioeconomic/docs/LearningSociety_WhitePaper.pdf

Coll, C. (1996). Constructivismo y educación escolar: ni hablamos siempre de lo mismo ni lo hacemos siempre desde la misma perspectiva epistemológica. Anuario de Psicología(69), 153-178. Obtenido de https://revistes.ub.edu/index.php/Anuario-psicologia/article/view/9094/11604

Ertmer, P., \& Newby, T. (1993). Conductismo, cognitivismo y constructivismo: una comparación de los aspectos críticos desde la perspectiva del diseño de instrucción. Performance Improvement Quarterly, 6(4), 50-72. Obtenido de https://www.galileo.edu/faced/files/2011/05/1. -ConductismoCognositivismo-yConstructivismo.pdf

Figueroa, H., Muñoz, K., Lozano, E., \& Zavala, D. (2017). Análisis crítico del conductismo y constructivismo, como teorías de aprendizaje en educación. Órbita Pedagógica, 4(1), 1-12. Obtenido de http://www.refcale.uleam.edu.ec/index.php/enrevista/article/view/2312/1245

Gadotti, M. (2003). Historia de las ideas pedagógicas. Buenos Aires: Siglo XXI editores Argentina.

Hergenhahn, B. (2011). introducción a la historia de la Psicología. México: Paraninfo.

Linares, A. (2007). Desarrollo cognitivo: las teorías de Piaget y Vygotsky. Obtenido de http://www.paidopsiquiatria.cat/files/teorias_desarrollo_cognitivo_0.pdf

Narvaja, P., \& Andrea, B. (2018). El problema de la pedagogía normativa. Digital Publisher CEIT, 3(3), 4-19. Obtenido de https://dialnet.unirioja.es/servlet/articulo?codigo=7143996

Peña, G. (2011). El conductismo: unas notas referenciales básicas. Analogías, 12, 7-20. Obtenido de http://revistasenlinea.saber.ucab.edu.ve/temas/index.php/analogias/article/view/4955/4119

Pløn Verhagen, B. (2006). Connectivism: a new learning theory? . Obtenido de https://es.scribd.com/doc/88324962/Connectivism-a-New-Learning-Theory 
Pons, J. (2013). ¿Hay vida más allá del cognitivismo? Encontrando respuestas en la psicología social. Información Psicológica(105), 110-129. doi:http://dx.medra.org/10.14635/IPSIC.2014.105.7

Rodríguez, A. (13 de julio de 2019). Conductismo en la educación: teoría conductista y ejemplos. Obtenido de Lifeder: https://www.lifeder.com/conductismo-en-la-educacion/

Siemens, G. (2005). Connectivism: A Learning Theory for the Digital Age. International Journal of Instructional Technology Distance Learning, 2(1), 1-9. Obtenido de http://www.itdl.org/Journal/Jan_05/index.htm

Soriano, J. (2011). Críticas al conductismo. Obtenido de http://aprendizajeycondicionamientouned.blogspot.com/2011/05/criticas-al-conductismo.html

Tumino, C., \& Bournissen, J. (2016). Conectivismo: Hacia El Nuevo Paradigma de la enseñanza por competencias. European Scientific Journal, 12(10), 112-128. doi:10.19044/esj.2016.v12n10p112

Valdez, F. (2012). Teorías educativas y su relación con las tecnologías de la información y de la comunicación (TIC). Obtenido de http://congreso.investiga.fca.unam.mx/docs/xvii/docs/L13.pdf

Zapata-Ros, M. (2014). Teorías y modelos sobre el aprendizaje en entornos. Obtenido de http://eprints.rclis.org/17463/1/bases_teoricas.pdf 